MEDINA IL; COILA VHC; GOMES CB; PEREIRA AS; NAZARENO NRX. 2014. Ocorrência de Meloidogyne ethiopica no Paraná e reação de cultivares de batata ao nematoide das galhas. Horticultura Brasileira 32: 482-485. DOI - http://dx.doi.org/10.1590/S0102-053620140000400018

\title{
Ocorrência de Meloidogyne ethiopica no Paraná e reação de cultivares de batata ao nematoide das galhas
}

\author{
Israel L Medina ${ }^{1}$; Víctor HC Coila ${ }^{1}$; Cesar B Gomes ${ }^{2}$; Arione S Pereira ${ }^{2}$; Nilceu RX Nazareno ${ }^{3}$ \\ ${ }^{1} U F P e l$, C. Postal 354, 96001-970 Pelotas-RS; islimes@hotmail.com; victorhugoc80@hotmail.com; ${ }^{2}$ Embrapa Clima Temperado C. \\ Postal 403, 96001-970 Pelotas-RS; cesar.gomes@embrapa.br; arione.pereira@embrapa.br; ${ }^{3}$ IAPAR, R. Maximo João Kopp 274, Santa \\ Candida, 80630-900 Curitiba-PR; nilceu@iapar.br
}

\begin{abstract}
RESUMO
Plantas de batata das cultivares Agata e Caesar debilitadas, com sistema radicular repleto de galhas e empipocamento severo nos tubérculos causado pelo nematoide das galhas (Meloidogyne sp.), foram detectadas em Contenda, município produtor tradicional do estado do Paraná. Posteriormente, fêmeas adultas do nematoide foram extraídas das raízes e tubérculos e submetidas à eletroforese identificando-se Meloidogyne ethiopica (Est E3) pelo perfil das bandas esterásticas reveladas. Utilizando-se uma população pura de $M$. ethiopica, plantas de batata de diferentes genótipos, mantidas em casa de vegetação em vasos contendo solo esterilizado, foram inoculadas com 5.000 ovos + juvenis de $2^{\underline{o}}$ estádio do nematoide por planta. Decorridos 55 dias da inoculação, cada planta foi avaliada quanto ao número de galhas e número de ovos, determinando-se o fator de reprodução do nematoide (FR) nos diferentes materiais testados. A cultivar Eliza comportou-se como moderadamente resistente a $M$. ethiopica; 'Ana', 'Bel', 'Agata' e 'Catucha', como moderadamente suscetíveis; 'Clara', 'Asterix', 'Cris' e 'Cota', suscetíveis (FR>4,00); e 'Caesar', como altamente suscetível.
\end{abstract}

Palavras-chave: Solanum tuberosum, detecção,resistência, fitonematoide.

\begin{abstract}
Meloidogyne ethiopica report in Parana state, Brazil, and reaction of potato cultivars to root-knot nematode

Potato plants of cultivars Agata and Caesar attacked by root-knot nematode (Meloidogyne sp.) showing root systems with multiple galls and protuberances in tubers were detected in Contenda, municipality traditionally producer of the Paraná state. Adult females of Meloidogyne sp. extracted from the potato roots and tubers and submitted to electrophoresis showed esterastic band profile as Meloidogyne ethiopica (Est E3). Using a pure population of $M$. ethiopica, potato plants of different cultivars, kept in pots with sterilized soil in greenhouse, were inoculated with 5,000 eggs + second stage juvenile of the nematode. Fifty-five days after the inoculation, each plant was evaluated by counting the number of galls and eggs, and determined the nematode reproduction factor (RF) in the different tested genotypes. The cultivar Eliza behaved as moderately resistant to M. ethiopica; 'Ana', 'Bel', 'Agata', and 'Catucha', as moderately susceptible; 'Clara', 'Asterix', 'Cris', and 'Cota', susceptible; and 'Caesar', as highly susceptible.
\end{abstract}

Keywords: Solanum tuberosum, report, resistance, root knot nematode.

(Recebido para publicação em 3 de fevereiro de 2014; aceito em 13 de agosto de 2014)

(Received on February 3, 2014; accepted on August 13, 2014)

\begin{abstract}
A bataticultura, no Brasil, ocupa uma área de 140.231 ha, sendo a produção estimada em 3.740.000 t (IBGE, 2012), cujo cultivo concentra-se principalmente, nas regiões Sul, Sudeste e Centro Oeste do País (Zambolin, 2011).

Dentre os problemas fitossanitários que limitam a produção da batata, os fitonematoides são responsáveis por causarem prejuízos significativos na cultura (Nazareno \& Gomes, 2010). As perdas mundiais provocadas pelos nematoides na cultura da batata são estimadas em 12,2\% (Sasser \& Freckman, 1987). No Brasil, são estimadas perdas de até $20 \%$ na produtividade, podendo esses prejuízos serem maiores devido
\end{abstract}

ao descarte de tubérculos com sintomas visíveis (Gomes \& Souza, 2003; Silva $\&$ Santos, 2007).

Considerando-se os fitonematoides de maior importância para a cultura da batata, o nematoide das galhas (Meloidogyne spp.) é considerado o mais patogênico. Este patógeno, ao penetrar no sistema radicular da planta, pode induzir a formação de galhas nas raízes, afetar negativamente o desenvolvimento da planta em função da menor absorção de água e nutrientes e, ainda, causar o empipocamento dos tubérculos, depreciando-os comercialmente (Gomes $\&$ Souza, 2003). Além disso, a planta afetada pelo nematoide pode tornar-se suscetível a outros patógenos, resultando em danos mais intensos (Silva \& Santos, 2007; Pinheiro \& Lopes, 2011).

Dentre as espécies do nematoide das galhas relacionadas à cultura da batata, no Brasil, registra-se a ocorrência de $M$. incognita, $M$. javanica, $M$. arenaria e $M$. hapla, sendo as três primeiras mais adaptadas a climas tropicais e subtropicais, e a última, a locais de clima mais ameno e temperaturas mais baixas (Charchar, 2001; Pinheiro \& Lopes, 2011).

Nos últimos anos, o aumento da área cultivada com batata no País, tem exposto a cultura a outras doenças de etiologias diversas como é o caso da 
pinta preta, causada por Alternaria grandis (Rodrigues \& Mizubuti, 2009), e da sarna prateada (Helminthosporium solani) (Nazareno, 2007). Dentre outros patógenos com potencial de dano na cultura, espécies do nematoide das galhas como M. ethiopica (Whitehead, 1969) tem sido relacionada a danos severos em espécies frutíferas, hortaliças e culturas anuais em diferentes regiões do Brasil (Carneiro et al., 2003; Castro et al., 2003; Carneiro et al., 2004; Gomes et al., 2005; Somavilla et al., 2011). No entanto, apesar de atacar plantas de batata (Whitehead, 1969), até o momento não há relatos de ocorrência em $S$. tuberosum no País.

De acordo com Charchar \& Moita (2001), a realização de um adequado manejo de nematoides fitoparasitas, impactantes à bataticultura, depende principalmente da cultivar, da época de plantio e do nível de infestação do solo onde a cultura será estabelecida. A rotação de culturas com espécies não hospedeiras de Meloidogyne spp. e o controle químico são as medidas de controle mais utilizadas no manejo dos nematoides das galhas em batata. Porém, os nematicidas são altamente tóxicos e têm a capacidade de acumular resíduos nos tubérculos quando aplicados na amontoa (Charchar, 1995). Além disso, espécies vegetais resistentes ou tolerantes a Meloidogyne spp, muitas vezes não são utilizadas devido a problemas de adaptação às condições edafoclimáticas do local ou pelo baixo retorno econômico (Carneiro, 1992).

O uso de cultivares de batata resistentes a Meloidogyne spp. é uma das alternativas mais desejadas por ser de custo mais baixo e eficientes. Porém, no Brasil há carência de material resistentes disponível no mercado e ainda, dispõe-se de pouca informação sobre a resistência de muitos dos genótipos cultivados às diferentes espécies do nematoide das galhas que ocorrem nas regiões produtoras do país. Dessa forma, o objetivo desse trabalho foi relatar a ocorrência de $M$. ethiopica em batata no estado do Paraná, além de avaliar a reação de diferentes genótipos comerciais a esta espécie de nematoide das galhas.

\section{MATERIAL E MÉTODOS}

Relato e identificação do nematoide das galhas em batata - Plantas de batata com raízes e tubérculos infectados pelo nematoide das galhas foram detectadas e coletadas em lavoura comercial do município de Contenda, região metropolitana de Curitiba-PR. No momento da coleta (março/2011), plantas das cultivares Caesar e Agata apresentavam-se debilitadas e exibiam sintomas de redução do crescimento, sistema radicular repleto de galhas e empipocamento severo nos tubérculos de batata (Figura 1).

A seguir, amostras de raízes e tubérculos de ambas as cultivares foram levadas ao laboratório de Fitopatologia da Embrapa Clima Temperado, para processamento (Hussey \& Barker, 1973) e quantificação do número de nematoides $/ 10 \mathrm{~g}$ de raízes. Posteriormente, fêmeas do nematoide das galhas (Meloidogyne sp.) de coloração branco leitosa, foram extraídas do restante das raízes e tubérculos das cultivares Caesar e Agata, e, imediatamente, foram submetidas à eletroforese para identificação da(s) espécie(s) pelo polimorfismo das bandas esterásticas em gel de poliacrilamida a 6\% (Carneiro \& Almeida, 2001).

Reação de genótipos de batata a M. ethiopica - Seis cultivares de batata (Eliza, Ana, Catucha, Cota, Clara e Bel) do programa de melhoramento da Embrapa Clima Temperado e parceiros, uma cultivar do programa do Iapar (cv. IPR Cris) e três cultivares comerciais importadas (Agata, Asterix e Caesar) foram avaliadas quanto à reação a $M$. ethiopica, em casa de vegetação,à temperatura de $24 \pm 5^{\circ} \mathrm{C}$.

Plantas individuais das diferentes cultivares, mantidas em vasos com $3 \mathrm{~kg}$ de solo esterilizado, foram inoculadas com 5.000 ovos + juvenis de segundo estádio (J2) de uma população pura de $M$. ethiopica (Carneiro \& Almeida, 2001), proveniente de batata (Contenda-PR) e mantidas em tomateiro (Solanum lycopersicum) 'Santa Cruz', em casa de vegetação. O experimento foi conduzido em delineamento inteiramente casualizado com seis repetições/cultivar. Como padrão de suscetibilidade e para confirmar a viabilidade do inóculo foram utilizadas plantas de tomate 'Santa Cruz’, que receberam o mesmo nível de inóculo do nematoide.

Cinquenta e cinco dias após a inoculação, as raízes de cada planta foram separadas da parte aérea, lavadas e avaliadas quanto ao número de galhas. A seguir, realizou-se a extração de ovos das raízes (Hussey \& Barker, 1973), para determinação da população final do nematoide e o cálculo do respectivo fator de reprodução (FR) de $M$. ethiopica ( $\mathrm{FR}=$ população final/população inicial), nas diferentes cultivares testadas (Oostenbrink, 1966). Para análise estatística, os valores de número de galhas (transformados em $\sqrt{x+1}$ ) e de FR, foram submetidos à ANOVA, sendo as médias dos tratamentos agrupadas pelo teste de agrupamento Scott-Knott (1974), a 5\% de probabilidade do erro. Foram consideradas como resistentes, as cultivares que apresentaram $\mathrm{FR}<1,00$; e suscetíveis, as que apresentaram FR $>1,00$.

\section{RESULTADOS E DISCUSSÃO}

Identificação e quantificação do nematoide das galhas em batata Utilizando-se a técnica de eletroforese, identificou-se $M$. ethiopica com fenótipo de esterase E3 (Rm: 0,92; 1,1 e 1,25) nas amostras das duas cultivares de batata onde o nematoide foi detectado. Da mesma forma, foi constatado elevado nível populacional de $M$. ethiopica no sistema radicular das plantas amostradas, variando de 830 a 1230 ovos $+\mathrm{J} 2$ do nematoide/ $10 \mathrm{~g}$ de raízes nas cultivares Agata e Caesar, respectivamente.

Reação das cultivares de batata a M. ethiopica - Os maiores valores de número de galhas foram observados em 'Caesar' seguido de 'Asterix' (Tabela 1). Quando avaliada a resistência das diferentes cultivares ao nematoide, observou-se que todas as cultivares foram suscetíveis ao nematoide comparativamente à testemunha (Tabela 1 ), no entanto o FR de $M$. ethiopica variou de 1,07 a 39,73, indicando variabilidade na taxa de reprodução em função do material testado.

Os elevados níveis de $M$. ethiopica detectados e os danos observados tanto nas raízes como nos tubérculos de batata, demonstram a suscetibilidade da 


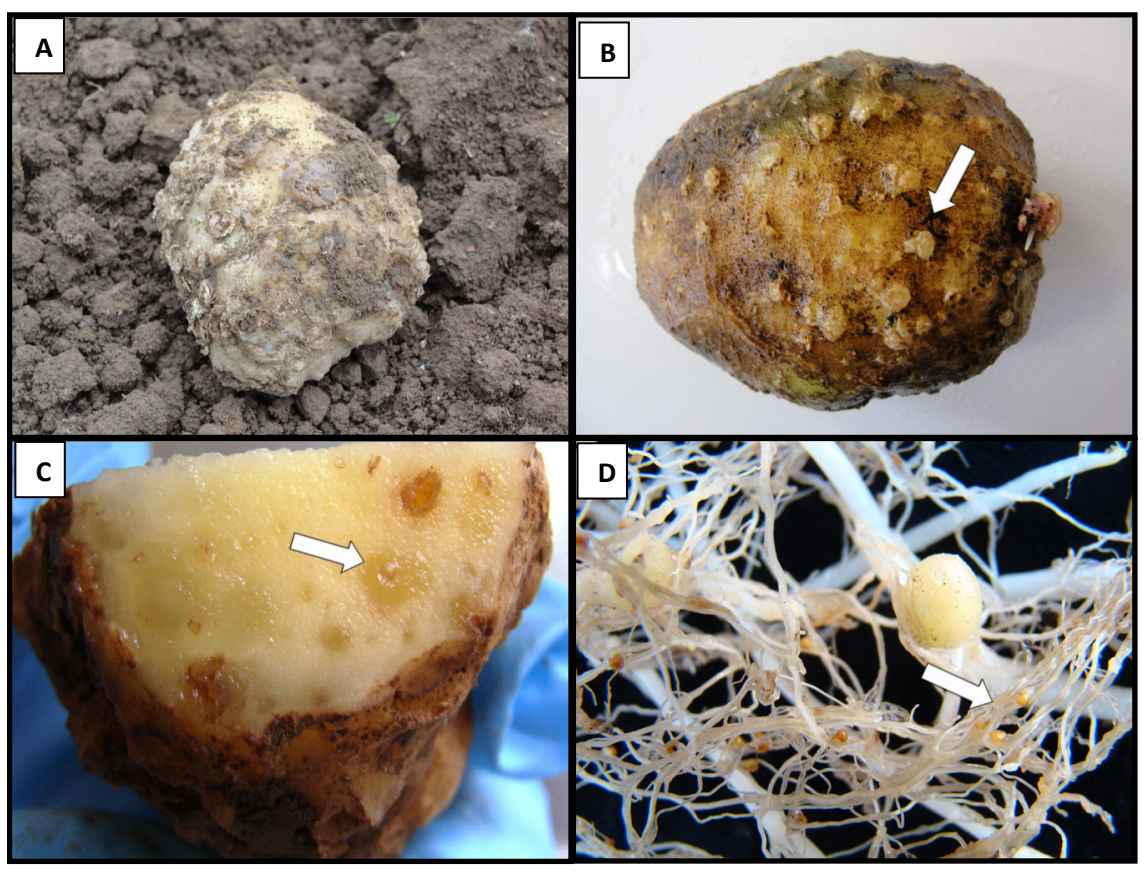

Figura 1. Sintomas causados por M. ethiopica em tubérculos e raízes de plantas de batata cv. Caesar; A, B= empipocamento em tubérculos; $\mathrm{C}=$ fêmea de $M$. ethiopica no interior do tubérculo; $\mathrm{D}=$ galhas e massas de ovos nas raízes (symptoms caused by $M$. ethiopica on tubers and roots of potato plants cv. Caesar; $\mathrm{A}, \mathrm{B}=$ potato tuber with galls; $\mathrm{C}=$ M. ethiopica females inside the tuber; $\mathrm{D}=$ galls and egg masses in the roots). Pelotas, Embrapa Clima Temperado, 2013.

Tabela 1. Reação de genótipos de batata a Meloidogyne ethiopica (reaction of potato genotypes to Meloidogyne ethiopica). Pelotas, Embrapa Clima Temperado, 2013.

\begin{tabular}{lccc}
\hline \multirow{2}{*}{ Genótipos } & \multicolumn{3}{c}{ M. ethiopica } \\
\cline { 2 - 4 } & Número de galhas & FR & Reação \\
\hline 'Santa Cruz' & $437,00 *$ & 27,18 & $\mathrm{~S}$ \\
Eliza & $18,66 \mathrm{c}$ & $1,07 \mathrm{e}$ & $\mathrm{S}$ \\
Agata & $27,83 \mathrm{c}$ & $2,22 \mathrm{~d}$ & $\mathrm{~S}$ \\
Catucha & $32,00 \mathrm{c}$ & $2,42 \mathrm{~d}$ & $\mathrm{~S}$ \\
Ana & $23,16 \mathrm{c}$ & $3,25 \mathrm{~d}$ & $\mathrm{~S}$ \\
Bel & $55,33 \mathrm{c}$ & $3,06 \mathrm{c}$ & $\mathrm{S}$ \\
Cris & $36,66 \mathrm{c}$ & $4,39 \mathrm{c}$ & $\mathrm{S}$ \\
Clara & $41,16 \mathrm{c}$ & $4,99 \mathrm{c}$ & $\mathrm{S}$ \\
Cota & $38,33 \mathrm{c}$ & $4,81 \mathrm{c}$ & $\mathrm{S}$ \\
Asterix & $130,33 \mathrm{~b}$ & $13,29 \mathrm{~b}$ & $\mathrm{~S}$ \\
Caesar & $907,00 \mathrm{a}$ & $39,73 \mathrm{a}$ & $\mathrm{S}$ \\
\hline CV $(\%)$ & 24,58 & 25,52 & \\
\hline
\end{tabular}

*Médias seguidas pela mesma letra na coluna pertencem ao mesmo agrupamento, pelo Teste de Scott-Knott, a 1\% de probabilidade; $\mathrm{R}=$ resistente; $\mathrm{S}=$ suscetível; ${ }^{1}$ Testemunha suscetível; $\mathrm{FR}=$ fator de reprodução (população final/população inicial de acordo com Oostenbrink, 1966); Foram consideradas como resistentes (R), as cultivares onde Meloidogyne ethiopica apresentou $\mathrm{FR}<1,00$; e, suscetíveis (S), FR $>1,00$, respectivamente [means followed by same letters in the column, pertain to the same grouping, Scott-Knott test, 1\%; R= resistant; $\mathrm{S}=$ susceptible; ${ }^{1}$ Susceptible control; $\mathrm{FR}=$ reproduction factor (final population/initial population, according to Oostenbrink, 1966); we considered as resistant (R) the cvs. for which Meloidogyne ethiopica presented FR $<1.00$ and susceptible (S) for values FR $>1.00$, respectively].

cultura a essa espécie de nematoide das galhas. Embora a batata seja relacionada como hospedeira de $M$. ethiopica há mais de 40 anos (Whitehead, 1969), este é o primeiro relato na cultura fora do Continente Africano.

No Brasil, o primeiro registro de ocorrência desse nematoide foi rela- tado na Serra Gaúcha em plantas de quivi (Actinidia deliciosa) (Carneiro et al., 2003). A seguir, M. ethiopica foi reportado em diferentes culturas de importância agrícola como a soja (Glycine max) em São Paulo (Castro et al., 2003), yacon (Polymnia sonchifolia) e tomate (Lycopersicum esculentum) no Distrito Federal (Carneiro et al., 2004), e em fumo (Nicotiana tabacum) no Rio Grande do Sul (Gomes et al., 2005). Apesar de poucos relatos sobre a distribuição e patogenicidade deste nematoide, danos significativos desta espécie parecem ser cada vez mais expressivos. Em países da América do Sul como o Chile (Magunacelaya, 2005; Carneiro et al., 2007), M. ethiopica tem sido associado ao declínio da videira e à ocorrência em quivi e tomateiro. Além disso, essa espécie foi detectada recentemente em aspargo (Asparagus officinalis), no Peru (Murga-Gutierrez et al., 2012), o que reforça a necessidade de se investigar os danos que possa causar em culturas adaptadas a regiões tropicais, subtropicais e temperadas, a exemplo da batata. Tais evidências vão de encontro ao fato de que a maioria das informações sobre a resistência da batateira ao nematoide das galhas, em condições brasileiras, está relacionada a M. javanica e M. incognita, espécies de maior ocorrência no país (Silva, 2009; Lima-Medina, 2013).

Embora a maioria das cultivares de batata testadas no presente trabalho tenha sido suscetível a $M$. ethiopica, 'Eliza', 'Catucha', 'Ana', 'Bel' e 'Agata' apresentaram algum nível de resistência, o que poderia viabilizar o cultivo desses materiais em áreas infestadas após a rotação com outras culturas hospedeiras em locais infestados com o nematoide. Além disso, 'Eliza' e 'Catucha' apresentam resistência moderada à requeima (Phytophthora infestans) (Gomes et al., 2005) e à pinta preta (Alternaria solani) (Pereira, 2010), características importantes dentro do sistema de produção.

Vale ressaltar que Meloidogyne ethiopica foi inserida na lista de alerta da EPPO (Reporting Service), desde 2011. Sendo assim, este nematoide requer maior atenção devido às infestações que podem ocorrer em espécies vegetais de considerada importância agrícola para 
o País. Dessa maneira, é importante incluir essa espécie de Meloidogyne em projetos de melhoramento visando à seleção de materiais resistentes tanto na cultura de batata como em outras hortaliças, culturas anuais e fruteiras estrategicamente importantes para a economia e sustentabilidade da agricultura brasileira.

\section{REFERÊNCIAS}

CARNEIRO RMDG. 1992. Princípios e tendências do controle biológico de nematoides com fungos nematófagos. Pesquisa Agropecuária Brasileira 27: 113-121.

CARNEIRO RMDG; ALMEIDA MRA. 2001. Técnica de eletroforese usada no estudo de enzimas dos nematoides das galhas para identificação de espécies. Nematologia Brasileira 25: 35-44.

CARNEIRO RMDG; ALMEIDA MRA; COFCEWICZ ET; MAGUNACELAYA JC; ABALLAY E. 2007. Meloidogyne ethiopica, a major root-knot nematode parasitising Vitis vinifera and other crops in Chile. Nematology 9: 635-641.

CARNEIRO RMDG; GOMES CB; ALMEIDA MR; GOMES ACC; MARTINS I. 2003. Primeiro registro de Meloidogyne ethiopica $\mathrm{em}$ plantas quivi no Brasil e reação em diferentes plantas hospedeiras. Nematologia Brasileira 27: $152-158$.

CARNEIRO RMDG; TIGANO MS; ALMEIDA MRA; SARAH JL. 2004. Identification and genetic diversity of Meloidogyne spp. on coffee from Brazil, Central America and Hawaii. Nematology 6: 287-298.

CASTRO JMC; LIMA R; CARNEIRO RMDC. 2003. Variabilidade isoenzimática de populações de Meloidogyne spp. proveniente de regiões brasileiras produtoras de soja. Nematologia Brasileira 27: 1-12.

CHARCHARJM. 1995. Meloidogyne em hortaliças. In: CONGRESSO INTERNACIONAL
DE NEMATOLOGIA TROPICAL, 27. CONGRESSO DA SOCIEDADE BRASILEIRA DE NEMATOLOGIA, 19. ENCONTRO ANUAL DA ORGANIZAÇÃO DOS NEMATOLOGISTAS DA AMERICA TROPICAL, 27. Programa e Anais... Rio Quente: SBN/ONTA. p. 149-153.

CHARCHAR JMM. 2001. Ciclo de vida de Meloidogyne spp. em batata. Gama: Embrapa Hortaliças, Boletim de Pesquisa e Desenvolvimento. $20 \mathrm{p}$.

CHARCHAR JM; MOITAAW. 2001. Resistência de genótipos de batata a Meloidogyne javanica. Pesquisa Agropecuária Brasileira 36: 535-540.

GOMES CB; CARBONARI JJ; MEDINA IL.; LIMA DL. 2005. Levantamento de Meloidogyne ethiopica em viveiros de quivi no Rio Grande do Sul e registro da ocorrência em fumo (Nicotiana tabacum) e guanxuma (Sida rhombifolia). CONGRESSO BRASILEIRO DE NEMATOLOGIA, 25. Anais... Piracicaba: CNB. p. 69.

GOMES CB; SOUZA RM. 2003. Doenças causadas por nematoides, In: $O$ cultivo da batata na região Sul do Brasil. Pelotas: Embrapa Clima Temperado, p. 321-349.

HUSSEY RS; BARKER KB. 1973. A comparison of methods of collecting inocula for Meloidogyne spp. including a new technique. Plant Disease 57: 1025-1028.

IBGE. 2012. ANUÁRIO ESTATÍSTICO DO BRASIL. 500p.

LIMA-MEDINA I. 2013. Diversidade de populações de Meloidogyne spp. e Pratylenchus spp. de diferentes regiões do sul do Brasil produtoras de batata e estudo da patogenicidade em Solanum spp. Pelotas: UFPEL. 117p. (Tese doutorado).

MAGUNACELAYA JC. 2005. Meloidogyne ethiopica y el cultivo de la vid en Chile. In: CONGRESSO BRASILEIRO DE NEMATOLOGIA, 25. Anais... Piracicaba: ESALQ/USP, SOB (CD-ROM).

MURGA-GUTIERREZ SN; COLAGIERO M; ROSSO LC; SIALER MMF. 2012. Ciancio A, Root-knot nematodes from asparagus and associated biological antagonists in Peru.
Nematropica 42: 57-62.

NAZARENO NRX. 2007. Sarna prateada da batata (Helminthosporium) Batata Show 7: 23-24.

NAZARENO NXR; GOMES CB. 2010. Doenças. In: PEREIRA AS. (org). Produção de batata no Rio Grande do Sul - Sistema de Produção, 19. Pelotas: Embrapa Clima Temperado. p. 55-68.

OOSTENBRINK M. 1966. Major characteristics of the relation between nematodes and plants. Wageningen: Landbouwhoge school 6: 1- 46.

PEREIRA AS. 2010. Produção de batata no Rio Grande do Sul - Sistema de Produção, 19. Pelotas-RS: Embrapa Clima Temperado. 95p.

PINHEIRO JB; LOPES CA. 2011. Manejo integrado de nematoides em cultivos de batata In: ZAMBOLIM L. (ed) Produção integrada da batata. v. 2. Viçosa: Universo Agrícola. p. 69-94.

RODRIGUES TTMS; MIZUBUTI ESG. 2009. Pinta preta: surge uma nova espécie. Batata Show 24: 14-16.

SASSER JN; FRECKMAN DW. 1987. A world perspective on nematology: the role of the society. In: VEECH JA; DISCKSON DW. (eds). Vistas on Nematology. Hyattsville: Society of Nematologist, p. 7-14.

SCOTT AJ; KNOTT M. 1974. A Cluster analysis method for grouping means in the analysis of variance. Biometrics 30: 507-512.

SILVA AR. 2009. Fitonematoides na cultura da batata: Reação de genótipos a Meloidogyne spp., distribuição de espécies e caracterização dos sintomas. Jaboticabal: UESP. 115p.

SILVA AR; SANTOS JM. 2007. Nematoides na cultura da batata no Brasil. 1. ${ }^{\text {a }}$. Ed, São Paulo: Associação Brasileira da Batata. 55p.

SOMAVILLA L; GOMES CB; CARNEIRO RMDG; CARBONARIJJ. 2011. Levantamento e caracterização de espécies do nematoide das galhas em quivi no Rio Grande do Sul. Tropical Plant Pathology 36: 89-94.

WHITEHEAD AG. 1969. The distribution of root-knot nematodes (Meloidogyne spp.) in Tropical Africa. Nematologica 15: 315-333.

ZAMBOLIM L. 2011. Produção integrada da batata Vol. I. Viçosa: Universo Agrícola. p. 438. 